\section{An Unusual Case of Gout in a Young Woman with Gitelman Syndrome}

\section{To the Editor:}

While gout is a very common and well-described disorder, it is rarely diagnosed in a young, premenopausal woman ${ }^{1}$. Overall, men are more than twice as likely to develop this condition over their lifetime as women. This sex difference is at its greatest in the 20- to 49-year age group, where incidence is $1.3 \%$ for men versus $0.4 \%$ for women ${ }^{2,3}$. The lower prevalence and incidence of gout in women has been attributed to the protective effects of estrogen and menstruation, which is consistent with the observation that it commonly manifests in women 10 to 15 years after menopause. When gout occurs in men under 30 years or in young women, investigation for a secondary cause is recommended ${ }^{4}$. This investigation should include checking for acquired risk factors and genetic disorders that exacerbate hyperuricemia (Table 1).

A 27-year-old woman was referred by a nephrologist for chronic and intermittent right first toe pain, redness, and swelling for the past year. It was exacerbated by tight shoes, and relieved with nonsteroidal antiinflammatory drugs (NSAID). She also reported several episodes of pain, swelling, and redness in both ankles over the past year. She was constitutionally well and denied fevers, weight loss, or night sweats. Further history revealed psoriasis $(\mathrm{PsO})$ treated with ultraviolet light therapy. She reported heavy alcohol use over the past 3 to 4 years, with binge drinking, and was previously hospitalized for acute pancreatitis. More recently she was diagnosed with Gitelman syndrome upon presenting with 2 years of episodically elevated creatinine along with hyponatremia, hypokalemia, elevated bicarbonate, and borderline high calcium. A diagnosis of Gitelman syndrome was made by exclusion of other conditions causing electrolyte imbalance and metabolic alkalosis, such as primary hyperaldosteronism, vomiting, and drugs (diuretics or laxatives). Her bland urinalysis, normal renal ultrasound, and no family history of renal disease further helped rule out glomerular, parenchymal, and other genetic causes. Her electrolyte abnormalities were managed with fluid and electrolyte replacement.

Given that Gitelman syndrome follows an autosomal recessive genetic pattern, it was hypothesized that both her parents were likely carriers. Confirmatory genetic testing was not performed for a number of reasons; the large number of involved genes and mutations, familial genetic heterogeneity, and high cost all limit the practicality of a molecular diagnostic approach ${ }^{5}$.

On examination, the patient was noted to be of a healthy body mass index. Musculoskeletal examination revealed 1 tender and swollen joint [right first metatarsophalangeal (MTP)] and restricted range of motion. The ankles were mildly restricted bilaterally, but no other joint swelling or tenderness was noted. Skin examination revealed psoriatic plaques over the elbows, knees, and shins.

On presentation to the rheumatology clinic, blood work revealed normal

Table 1. Secondary causes of hyperuricemia that should be considered in individuals with low risk for gout.

Acquired

- Increased nucleic acid turnover: leukemia, myeloma, radiotherapy, chemotherapy (tumor lysis syndrome)

- $\quad$ Altered ATP metabolism: alcohol abuse, excess fructose intake

- Enhanced tubular secretion or reabsorption of uric acid: dehydration, starvation, insulin resistance, competitive organic ions (lactate, acetoacetate)

- Drugs/toxins: salicylate, thiazide diuretics, lead poisoning

- Comorbidities: chronic kidney disease, obesity, hypertension Genetic

- Inherited metabolic disorders: Lesch-Nyhan Syndrome, glycogen storage disease

- Renal insufficiency because of genetic kidney defects: Gitelman syndrome, Bartter syndrome electrolytes and serum creatinine $83 \mu \mathrm{mol} / 1$; however, previous creatinine levels ranged from 66 to $194 \mu \mathrm{mol} / 1$. Uric acid (UA) was significantly elevated at $759 \mu \mathrm{mol} / 1$. Liver enzyme were abnormal: aspartate aminotransferase $110 \mathrm{U} / 1$, alanine aminotransferase $137 \mathrm{U} / 1$, alkaline phosphatase $172 \mathrm{U} / 1$, and $\gamma$-glutamyl transferase $91 \mathrm{U} / 1$.

Synovial fluid aspiration of the first MTP joint revealed chalky white material, with UA crystals on polarized microscopy. The joint was injected with corticosteroids and the patient was instructed to stop all alcohol intake and NSAID use. At followup 2 weeks later, she reported significant improvement in pain and swelling, and cessation of alcohol intake resulted in normalization of liver enzymes. Her UA remained elevated at $759 \mu \mathrm{mol} / 1$. Given the tophaceous gout tophus deposit and recurrent joint symptoms, UA-lowering therapy with febuxostat $80 \mathrm{mg}$ daily plus colchicine $0.6 \mathrm{mg}$ bid was initiated. One year later, she was asymptomatic with no recurrence of gouty flares, her UA level decreased to $141 \mu \mathrm{mol} / 1$, and creatinine was $65 \mu \mathrm{mol} / 1$

Our patient was diagnosed with Gitelman syndrome, a rare autosomal recessive inherited renal tubular defect (prevalence estimated at 1:40,000). The clinical presentation is known to mimic the effects of a thiazide diuretic with a defect in the thiazide-sensitive sodium chloride cotransporter (NCCT) in the renal distal convoluted tubule ${ }^{5,6}$. Sodium-chloride reabsorption is impaired leading to salt loss and reduced circulating volume, activating the renin-angiotensin-aldosterone system (RAAS) leading to hypokalemia and hypochloremic metabolic alkalosis ${ }^{7}$. Thiazide diuretics are known to increase serum UA levels ${ }^{8}$. Similarly, dietary salt restriction has been shown to increase uric acid concentrations, perhaps by altering renal tubular function ${ }^{9,10}$. However, to our knowledge there is no literature describing an association between Gitelman syndrome and gout, and therefore it is unclear what levels of serum UA would be expected in this syndrome.

Historically, it has been accepted that thiazide diuretics increase net UA reabsorption indirectly through diuretic-induced volume depletion and activation of the RAAS system ${ }^{8,11}$. More recent data suggest diuretics have a direct interaction on renal UA handling through the voltage-driven UA transporter hNPT4/SLC17A3 ${ }^{12}$, as well as the cyclic nucleotide transporter, $\mathrm{MRP} 4 / \mathrm{ABCC} 4^{13}$. Interestingly, Gitelman syndrome has recently been described as associated with mutations in the UA transporter gene, $S L C 17 A 3^{14}$. A detailed mechanism of thiazide diuretic effect on renal handling of UA is depicted in Figure 1. These findings along with clinical observations in our case report support a potential novel mechanism for the effect of Gitelman syndrome on tubular UA transport that would be the cause of its association with hyperuricemia.

Gout is most commonly seen in older men and postmenopausal women, and has well-described risk factors. It is uncharacteristic to observe tophaceous gout in a young woman, a clinical presentation that should prompt rheumatologists to investigate a secondary cause. In our patient, there were many contributing factors that drove elevated UA levels and clinical development of tophaceous gout. Her risk factors included the thiazide-like effect of Gitelman syndrome, along with excess alcohol use, acute renal failure, and increased cell turnover from PsO. She was managed with UA-lowering therapy and modification of lifestyle, including cessation of alcohol and NSAID use. The target level of UA was achieved, an important goal to prevent future joint symptoms and damage.

To our knowledge, our case study highlights for the first time the association between Gitelman syndrome and gout. We identified Gitelman syndrome as a novel risk factor for the development of gout and provided early insights into the pathogenesis of its association with hyperuricemia. However, further investigation is warranted into the pathogenesis of Gitelman syndrome, hyperuricemia, and this association.

Research ethics board approval was not required for the publication of this case report.

SARAH M. TROSTER, MD, Division of Rheumatology, St. Michael's Hospital, University of Toronto, Toronto, Ontario; JOSHUA E. RAIZMAN, PhD, Department of Pathology and Laboratory Medicine, University of Alberta Hospital, University of Alberta, Edmonton, Alberta;

Personal non-commercial use only. The Journal of Rheumatology Copyright $\odot$ 2016. All rights reserved. 


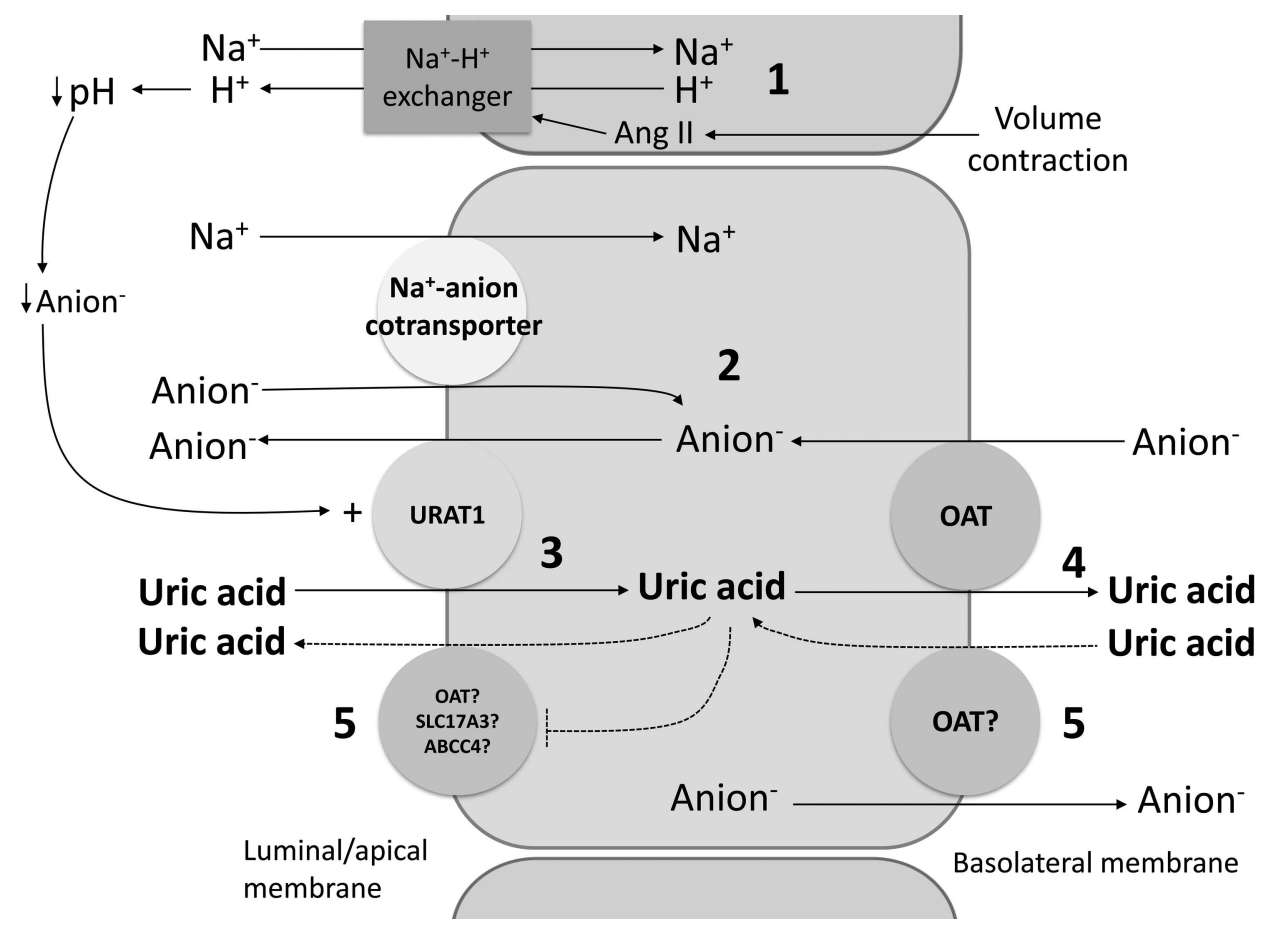

Figure 1. The effect of thiazide diuretic on uric acid transport in the renal proximal tubule. Most of the filtered uric acid is reabsorbed through the URAT1 located on the apical/luminal membrane of renal proximal tubular cells. It functions to transport uric acid from the proximal lumen in exchange for intracellular anions. It is theorized that thiazide diuretics increase uric acid reabsorption through volume contraction and activation of the RAAS system. (1) Ang II acts on the sodium-hydrogen ion transporter in the proximal tubule to increase sodium reabsorption. (2) There is sodium-dependent entry of monovalent anions (such as lactate, pyruvate, $\beta$-hydroxybutyrate, and acetoacetate) through the sodium-anion co-transporter that raises acidity in the renal tubule and increases intracellular anion concentration. (3) The increased intracellular anion concentration gradient drives reabsorption of uric acid through the URAT1. (4) OAT in the basolateral membrane transport uric acid into the renal capillaries by fueling anion exchange into the cell as it flows down its concentration gradient. (5) Through unclear mechanisms, OAT or other pathways are also involved in uric acid secretion by stimulating anion exchange into the proximal cell, or diuretic-induced direct inhibition of uric acid excretion by the uric acid transporters SLC17A3 and ABCC4. Continual stimulation of the URAT1 transporter and inhibition of secretion and excretion of uric acid leads to net reabsorption and in turn causes hyperuricemia. URAT1: urate transporter-1; Ang II: angiotensin II; OAT: organic anion transporters; RAAS: renin-angiotensin-aldosterone system.

LAURENCE RUBIN, MD, Division of Rheumatology, St. Michael's Hospital, University of Toronto, Toronto, Ontario, Canada. Address correspondence to Dr. L. Rubin, Division of Rheumatology, St. Michael's Hospital, Bond Wing 3-061, 30 Bond Street, Toronto, Ontario M5B 1W8, Canada.E-mail: rubin@smh.ca

\section{REFERENCES}

1. Kim KY, Ralph Schumacher H, Hunsche E, Wertheimer AI, Kong SX. A literature review of the epidemiology and treatment of acute gout. Clin Ther 2003;25:1593-617.

2. Kramer HM, Curhan G. The association between gout and nephrolithiasis: the National Health and Nutrition Examination Survey III, 1988-1994. Am J Kidney Dis 2002;40:37-42.

3. Lawrence RC, Felson DT, Helmick CG, Arnold LM, Choi H, Deyo RA, et al; National Arthritis Data Workgroup. Estimates of the prevalence of arthritis and other rheumatic conditions in the United States. Part II. Arthritis Rheum 2008;58:26-35.

4. Klippel JH. Primer on the rheumatic diseases. In: Choi HK, editor. Gout: epidemiology, pathology and pathogenesis, 13th ed. New York: Springer; 2009:250-7.

5. Naesens M, Steels P, Verberckmoes R, Vanrenterghem Y, Kuypers
D. Bartter's and Gitelman's syndromes: from gene to clinic. Nephron Physiol 2004;96:65-78.

6. Simon DB, Nelson-Williams C, Bia MJ, Ellison D, Karet FE, Molina AM, et al. Gitelman's variant of Bartter's syndrome, inherited hypokalaemic alkalosis, is caused by mutations in the thiazide-sensitive Na-Cl cotransporter. Nat Genet 1996;12:24-30.

7. Simon DB, Lifton RP. The molecular basis of inherited hypokalemic alkalosis: Bartter's and Gitelman's syndromes. Am J Physiol 1996;27:F961-6.

8. Carlsen JE, Køber L, Torp-Pedersen C, Johansen P. Relation between dose of bendrofluazide, antihypertensive effect, and adverse biochemical effects. BMJ 1990;300:975-8.

9. Egan BM, Weder AB, Petrin J, Hoffman RG. Neurohumoral and metabolic effects of short-term dietary $\mathrm{NaCl}$ restriction in men. Relationship to salt-sensitivity status. Am J Hypertens 1991; 4:416-21.

10. Ruppert M, Diehl J, Kolloch R, Overlack A, Kraft K, Göbel B, et al. Short-term dietary sodium restriction increases serum lipids and insulin in salt-sensitive and salt-resistant normotensive adults. Klin Wochenschr 1991;69 Suppl 25:51-7.

11. Choi HK, Mount DB, Reginato AM; American College of

$$
\text { Personal non-commercial use only. The Journal of Rheumatology Copyright @ } 2016 \text {. All rights reserved. }
$$


Physicians; American Physiological Society. Pathogenesis of gout. Ann Intern Med 2005;143:499-516.

12. Jutabha P, Anzai N, Kitamura K, Taniguchi A, Kaneko S, Yan K, et al. Human sodium phosphate transporter 4 (hNPT4/SLC17A3) as a common renal secretory pathway for drugs and urate. J Biol Chem 2010;285:35123-32.

13. El-Sheikh AA, van den Heuvel JJ, Koenderink JB, Russel FG.

Effect of hypouricaemic and hyperuricaemic drugs on the renal urate efflux transporter, multidrug resistance protein $4 . \mathrm{Br} \mathrm{J}$ Pharmacol 2008;155:1066-75.
14. Takeuchi Y, Mishima E, Shima H, Akiyama Y, Suzuki C, Suzuki T, et al. Exonic mutations in the SLC12A3 gene cause exon skipping and premature termination in Gitelman syndrome. J Am Soc Nephrol 2015;26:271-9.

J Rheumatol 2016;43:11; doi:10.3899/jrheum.160244 